Beatriz H. Juarez and Luis M. Liz-Marzán*

\title{
Microgels and Nanoparticles: Where Micro and Nano Go Hand in Hand
}

\begin{abstract}
The integration of different types of materials in a single hybrid system allows the combination of multiple functionalities, which can even be used in conjunction with each other. This strategy has been exploited in nanoscale systems for the creation of so-called smart nanomaterials. Within this category, the combination of inorganic nanoparticles with stimuli-responsive microgels is of very high interest because of the wide variety of potential applications. We present here a short overview of this type of materials in which the nano- and micro-scales get nicely integrated, with a great potential to expand the range of technological applications. We focus mainly on the integration of metal nanoparticles, either by themselves or in combination with semiconductor and magnetic nanoparticles. Various examples of the synergic properties that can be obtained are described, as well as the possibility to extract useful information when optical tweezers are used to manipulate single particles. We expect that this review will stimulate additional research in this field.
\end{abstract}

Keywords: Microgels, PNIPAM, Metal Nanoparticles, Quantum Dots, Optical Tweezers.

DOI 10.1515/zpch-2014-0578

Received August 7, 2014; accepted October 23, 2014

Dedicated to Horst Weller on the occasion of his $60^{\text {th }}$ birthday

\section{Introduction}

Out of a diverse palette of gels, those dispersible in water, i. e. hydrogels, have triggered numerous studies due to their ability to respond against changes of tem-

*Corresponding author: Luis M. Liz-Marzán, Bionanoplasmonics Laboratory, CIC biomaGUNE, Paseo de Miramón 182, 20009 Donostia - San Sebastian, Spain; and Ikerbasque, Basque Foundation for Science, 20009 Bilbao, Spain, e-mail: Ilizmarzan@cicbiomagune.es Beatriz H. Juarez: Universidad Autónoma de Madrid, Dpto. Química-Física Aplicada and IMDEA Nanoscience, Campus de Cantoblanco, 28049 Madrid, Spain 
perature, $\mathrm{pH}$, magnetic and/or electric fields, osmotic pressure, to detect specific molecules such as glucose or antigens, their properties as scaffolds to repair tissues, and their potential use as delivery systems for therapeutic or imaging agents [1-6]. In fact, their response to external stimuli has granted these materials the consideration of smart or intelligent gels. A hydrogel is defined as a network of hydrophilic polymer chains dispersed in water. Natural hydrogel materials include agarose, methylcellulose or hyaluronic acid. Among a wide variety of synthetic hydrogels, poly(N-isopropylacrylamide) (pNIPAM) has received special attention. pNIPAM is a temperature-responsive polymer that undergoes a reversible phase transition from a swollen hydrated state to a shrunken dehydrated state at $32^{\circ} \mathrm{C}$, which is thus its lower critical solution temperature (LCST) [1]. This means that around this temperature the interactions between chains in the polymeric network and the solvent experience a dramatic change, boosting their reorganization. For pNIPAM, as temperature rises hydrogen bonds connecting amine groups in the polymer weaken, enhancing polymer-polymer interactions, as sketched in Figure $1[7,8]$. This leads to expelling water (or other compounds previously embedded in the polymeric network interior) and changes the surface properties $[9,10]$. A distinction has been made between gels (or macrogels) and microgels, the latter term referring to the polymeric network as a colloidal dispersion in water, or simply to hydrogel microspheres. While macrogels and microgels share most of their physical-chemical properties, microgels exhibit a number of important additional features that derive from their colloidal nature: microgels are freeflowing liquids and the high surface to volume ratio favors rapid responses triggered by environmental changes, as well as mass transport from the inner core to the outer medium and vice versa [11, 12]. Furthermore, microgels can be assembled into 2D or 3D colloidal crystals with tunable optical properties [13].

Regarding synthetic routes, NIPAM is frequently polymerized in emulsions. Co-polymerization of NIPAM with other organic co-monomers such as acrylic acid, maleic acid or styrene can be used to induce changes in the swelling kinetics and thermodynamics, through variation of both the elastic properties and the osmotic pressure [1]. Other variations in the synthesis serve to adjust the water content within as much as 10 to $90 \mathrm{wt} \%$. Furthermore, the surface of microgels is usually negatively charged, which is the result of the initiators or other monomers susceptible of copolymerization. Cross-linking monomers such as N,N-methylenebisacrylamide (MBA) are regularly used and can also modify the microgel properties. The polymerization kinetics of other co-monomers with respect to those of NIPAM will influence the final properties of the colloid in terms of compositional gradient and/or surface charge. The large amount of scientific work reporting microgel research in the last decades evidences the high interest on these materials. 


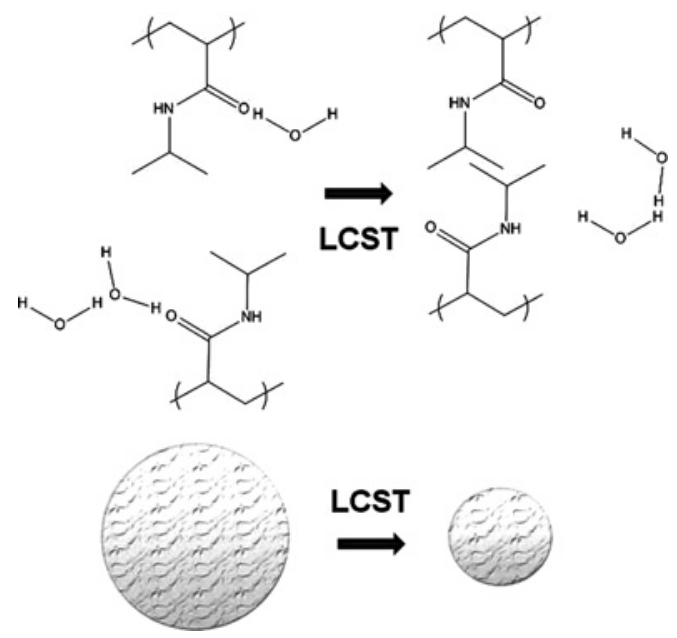

Figure 1: Sketch showing the structure of pNIPAM below and above its lower critical solution temperature (LCST). Hydrogen bonds break leading to reorganization of the polymer and the solvent, rendering the hydrophilic swollen polymer below LCST into a hydrophobic polymer in a shrunken state above LCST.

Some of the most important features of microgels in general and of pNIPAM in particular are related to the versatility to tune their properties, namely: (i) NIPAM can be copolymerized with one or more co-monomers to produce colloids with tunable LCST and shrinking properties, (ii) their surface charge and functionalization can be controlled during the synthesis, and (iii) core-shell-like structures can be produced, providing additional tunability of the physico-chemical properties, derived from the synergic combination of the properties of the core and the shell. Furthermore, out of core-shell structures, hollow microgel particles can be obtained by selectively dissolving the core $[14,15]$.

\section{2 pNIPAM microgels and nanoparticles}

The incorporation of metal, magnetic or semiconductor nanoparticles (NPs) within (pNIPAM) microgels is very attractive because the functionalities that are inherent to the NPs can be added or combined with those of the microgels. In this section we focus on the synthesis and characterization of hybrid systems combining pNIPAM microgels and NPs. From the synthetic point of view, NPs (i) can be added in-situ during the hydrogel synthesis to generate microgels loaded with NPs [16], (ii) can decorate the surface of previously prepared microgels [17] 

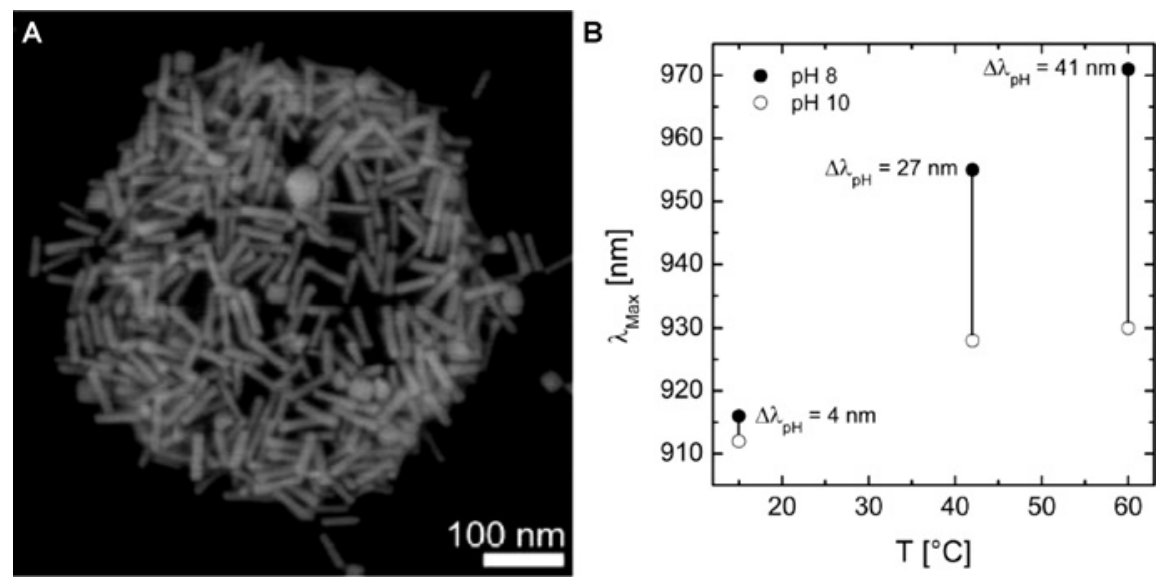

Figure 2: A) TEM image of pNIPAM beads decorated with Au nanorods. The inset shows a higher magnification image. B) Position of the longitudinal LSPR band during various swelling and collapse events, $n$, between $20^{\circ} \mathrm{C}$ and $40{ }^{\circ} \mathrm{C}$.

or (iii) can be used as seeds from which the gel can be grown as a corona/shell, NPs@pNIPAM [18].

Among other properties, metal nanoparticles have raised much interest within the context of the use of nanostructures as small sources of heat to locally increase the temperature. For example, laser beams can be used for the destruction of tumor tissues through hyperthermia, but this technique requires the development of materials that are susceptible to release heat upon optical excitation [19]. Metallic NPs absorb electromagnetic radiation and subsequently release energy as heat, thus increasing the temperature of the surrounding medium. The ability to transfer heat from optically excited metallic NPs has triggered numerous studies in which NPs can be used as remote controllers of the phase transition of pNIPAM microgels. Several groups have obtained hybrid microgels by either polymerizing NIPAM in the presence of metallic NPs or by simultaneous nucleation and growth of both components [20]. An example of decorated microgels is the adsorption of Au nanorods on the surface of pNIPAM microgels, as shown in Figure $2 \mathrm{~A}$. In this case, a polyelectrolyte was used to promote electrostatic attraction between the nanorods and the pNIPAM microbeads. It is known that localized surface plasmon resonances (LSPR) in metal NPs are strongly influenced by particle size and shape, as well as by the dielectric properties of the environment and interparticle interactions [21]. Au nanorods exhibit two well-differentiated plasmon modes, transverse and longitudinal LSPRs. When Au nanorods are adsorbed on pNIPAM microgels, the thermally induced collapse of the polymer network has 

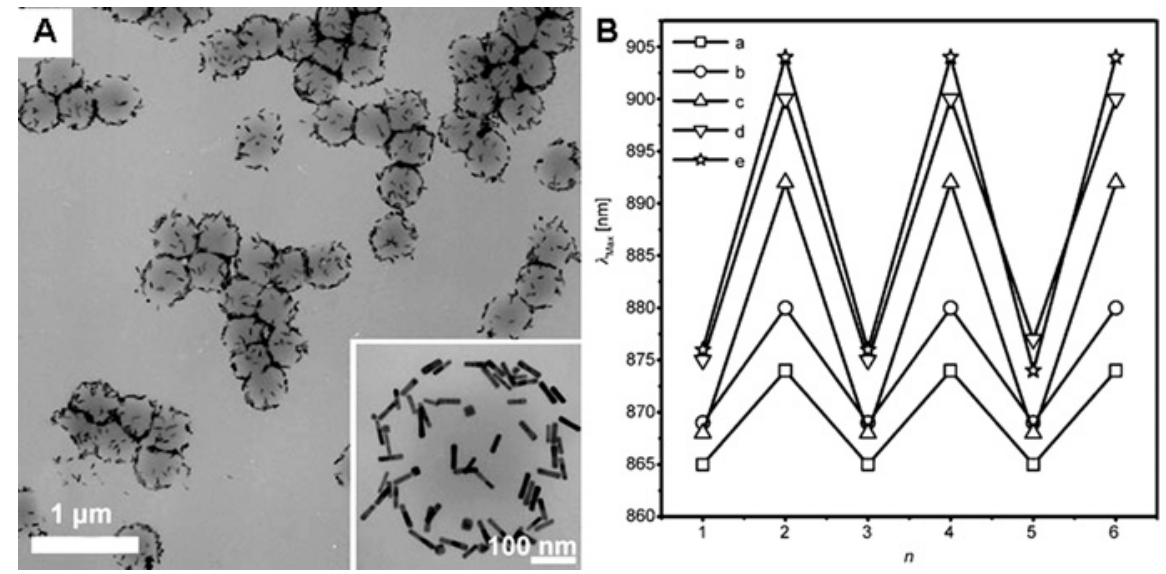

Figure 3: A) SEM image of an individual pNIPAM-co-AAA microgel highly loaded with $\mathrm{Au}$ nanorods. B) LSPR band positions $\left(\lambda_{\max }\right)$ as a function of $\mathrm{pH}$ and temperature. The filled symbols show values for the hybrid systems at $\mathrm{pH} 8$, while open ones were obtained at $\mathrm{pH} 10$. $\Delta \lambda_{\mathrm{pH}}$ is the plasmon shift between both $\mathrm{pH}$ values.

been found to lead to a fully reversible red shift of the longitudinal plasmon band of the nanorods (see Figure 2B) [22]. Such optical response is, in this case, the result of slight changes in the refractive index of the microgel which are recorded by the highly sensitive NPs. Copolymerization of NIPAM with allylacetic acid (AAA) yields a more interesting hybrid system (pNIPAM-co-AAA) [23], which is sensitive not only to temperature but also to $\mathrm{pH}$. Additionally, control over charge density (provided by the acid) allows for higher Au-nanorods coverage, as shown in Figure 3A. This hybrid system has been demonstrated to show not only a reversible 55 $\mathrm{nm}$ LSPR red shift with temperature, but also a $41 \mathrm{~nm}$ red shift when the $\mathrm{pH}$ varies between 8 and 10 (see Figure 3B). In this case, the larger red shift is the result of the plasmon coupling between neighboring NPs (in densely covered microgels) when the polymer collapses.

Au NPs can also be immobilized on the surface of pNIPAM microgels by electrostatic interactions between the capping ligands of the NPs and functional groups grafted to the polymeric network. An example is shown in Figure 4A, where spherical Au NPs capped with biocompatible polyethylene oxide (PEO) ligands [24] were used to decorate the surface of pNIPAM microbeads copolymerized with maleic acid, which renders the microgel surface with high negative charge at appropriate $\mathrm{pH}$ values (similar to NIPAM copolymerization with AAA, see above). Plasmon coupling can be clearly identified for this system in Figure 4B,D. For small enough distances between metal NPs, which are achieved upon heating densely covered pNIPAM microgels (see Figure 4C), additional plasmon-coupled 

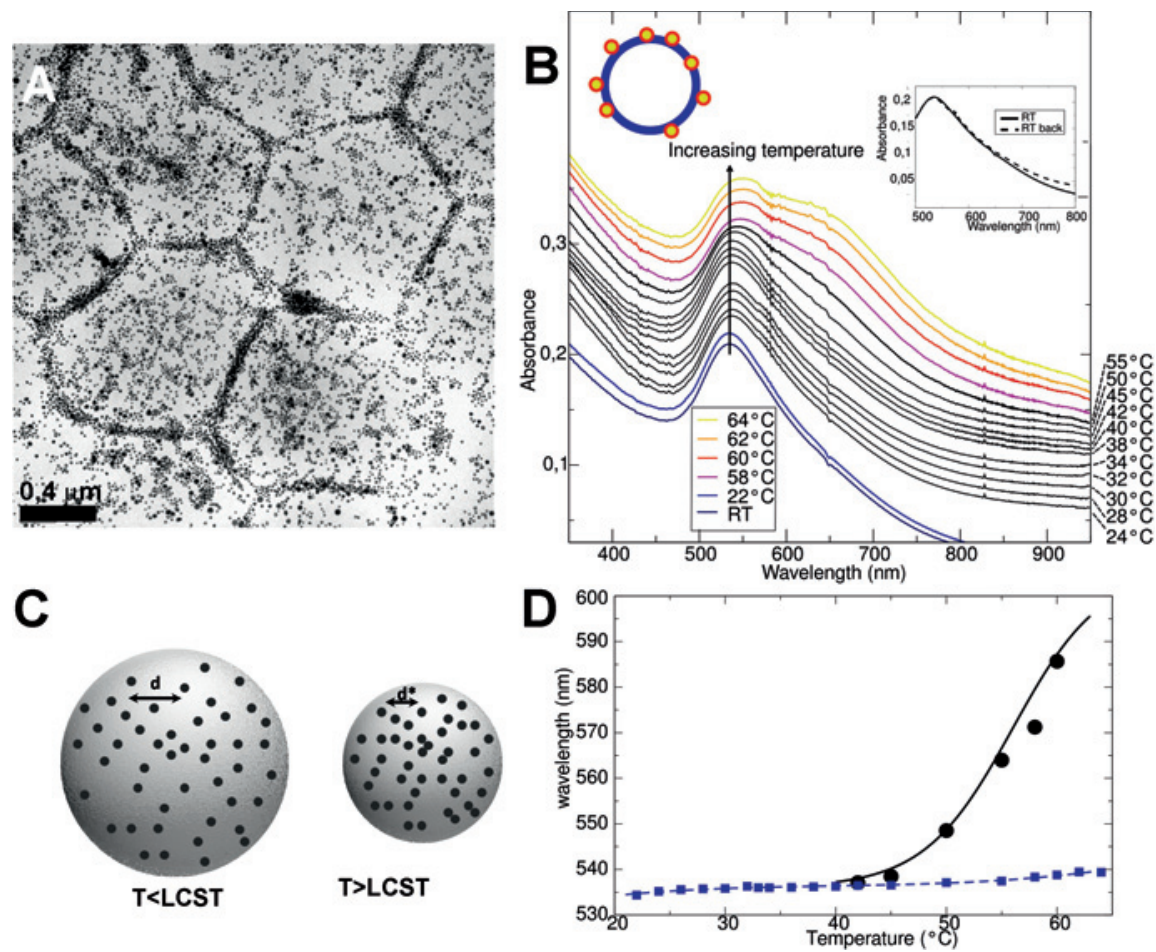

Figure 4: A) TEM image of pNIPAM microgels decorated with Au NPs capped with PEO-based ligands. B) Absorption spectra of densely covered Au-pNIPAM beads at different temperatures. Left inset: illustration of the decorated systems; Right Inset: Comparison of the initial spectrum with that obtained after cooling back to room temperature. C) Sketch showing the decrease of average NP distance upon reaching the LCST, where pNIPAM collapses. D) Evolution of fundamental (squares) and coupled plasmon (circles) modes with temperature for pNIPAM microgels highly covered with Au NPs. The lines are guides to the eye.

modes appear as a collective excitation of several NPs. Another possibility of combining Au NPs with pNIPAM comprises a single Au NP as the core, covered by a microgel shell (Au@pNIPAM). A number of synthetic approaches have been described, including priming the metal NP surface with styrene and divinylbenzene prior to NIPAM polymerization [18], as well as the use of molecules containing a vinyl skeleton with carboxylic acid or amine functional groups [25, 26]. Novel strategies include introducing in the outmost layer a polyelectrolyte containing the required vinyl functional groups [27].

Magnetic functionality (mainly via iron oxide NPs) has also been incorporated within pNIPAM microgels. This not only serves to manipulate the particles with external magnetic fields, but also provides heating capacity derived from the ex- 

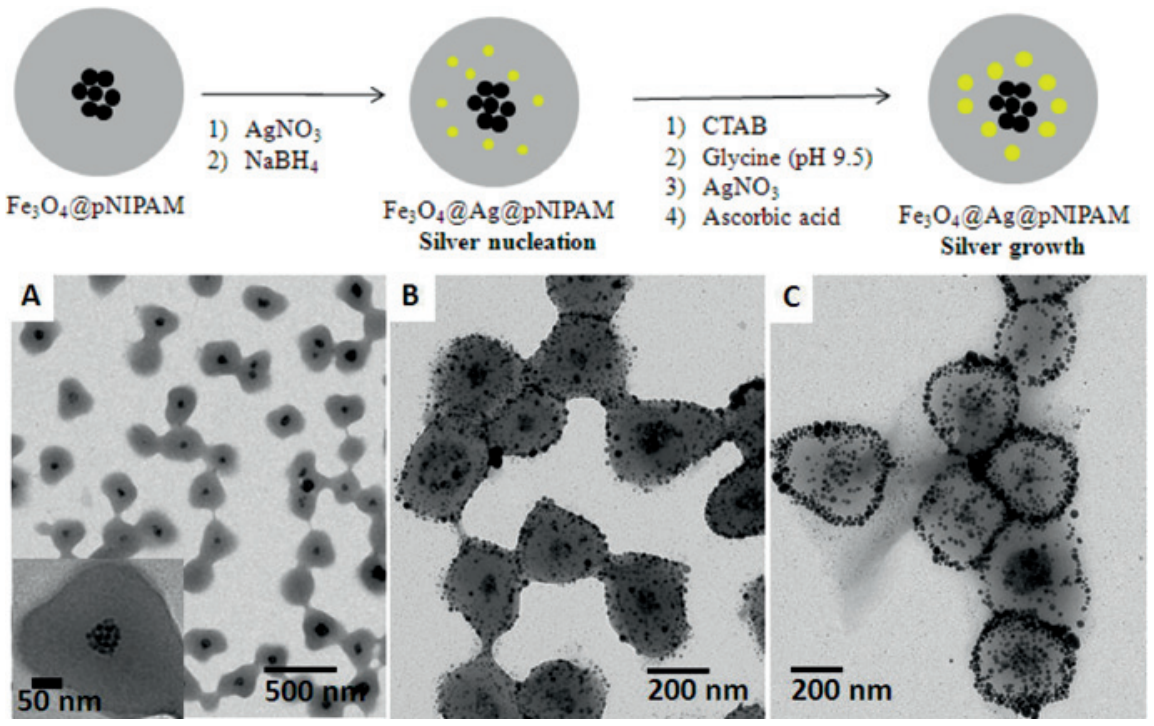

Figure 5: Sketch of the synthetic steps used to grow Ag NPs within the polymeric pNIPAM network covering magnetite NPs as shown in the TEM images (A). Small Ag NPs are formed in a first step by reducing $\mathrm{Ag}^{+}$with a strong reducing agent (B). The size of Ag NPs is increased by growing on the existing Ag seeds under softer reducing conditions (C) (see text for details).

citation of the magnetic NPs under alternating magnetic fields. The adsorption of magnetic iron oxide NPs on the surface of pNIPAM microgel beads copolymerized with styrene in a core-shell fashion has been reported [28]. Even more complex structures have been realized by growing a pNIPAM corona on single $\mathrm{Fe}_{2} \mathrm{O}_{3}$ covered with a sacrificial $\mathrm{SiO}_{2}$ shell [29], or through the combination of magnetic and metallic NPs covered within the same pNIPAM microgel beads [30]. The later structure is illustrated in Figure 5, where a sketch describes the synthetic steps and the results are shown in the TEM images below. In this case, magnetite $\mathrm{Fe}_{2} \mathrm{O}_{3}$ NPs were capped with butenoic acid [31], which facilitated the encapsulation with pNIPAM. For the nucleation of silver NPs in the interior of the $\mathrm{Fe}_{2} \mathrm{O}_{3}$ NPs @pNIPAM hybrid system, sodium borohydride was used to reduce silver cations distributed within the polymeric network. The size of the metal seeds could be increased by a further growth step employing a cationic surfactant and glycine. These hybrid systems show clear advantages because: (i) the collapse of the pNIPAM network above its LCST leads to a decrease in the average distance between Ag NPs inducing plasmon coupling and, (ii) the manipulation and concentration of the composites is possible due to the magnetic component. These combined functionalities have been exploited for sensing, as described below. 


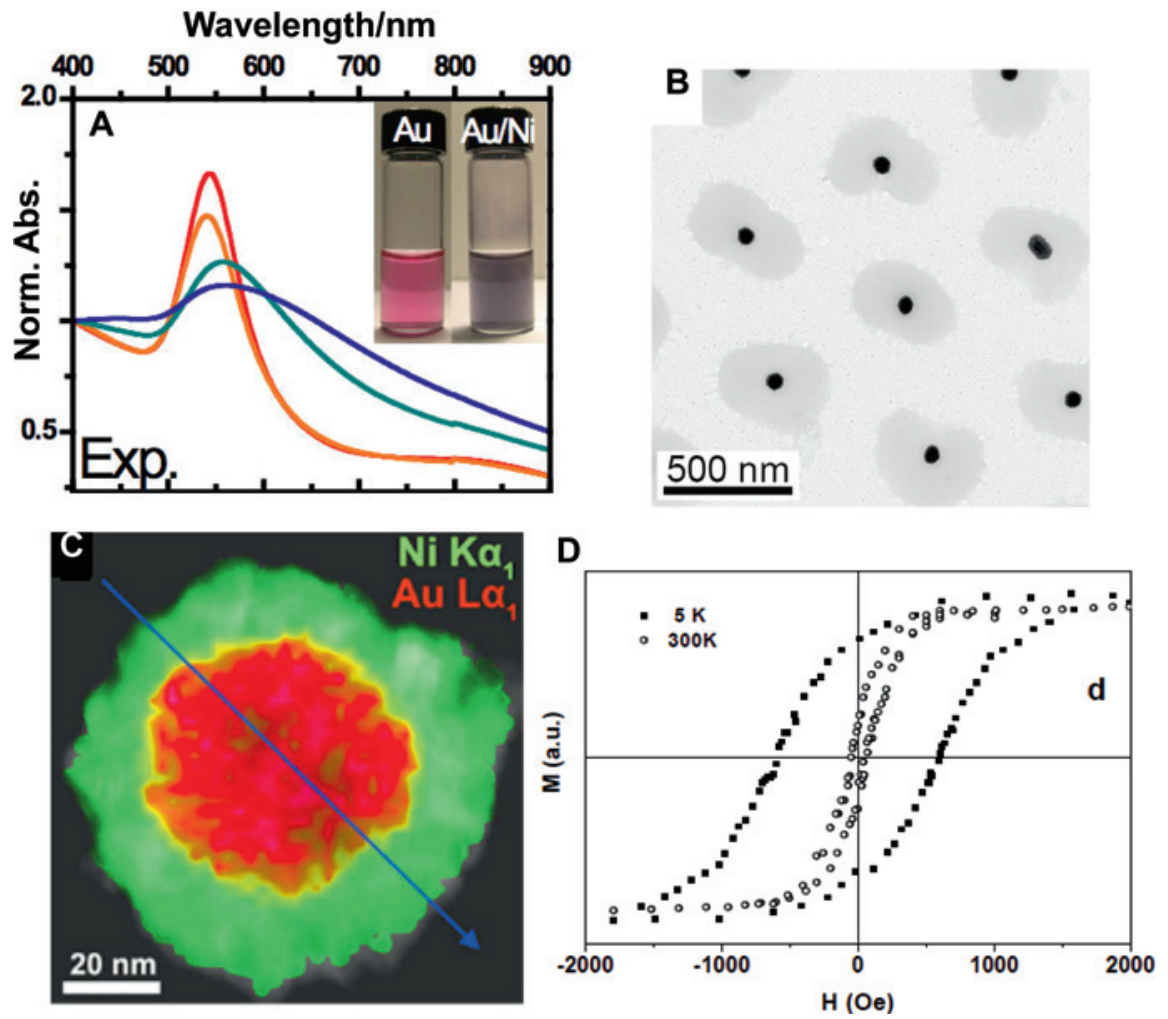

Figure 6: A) Optical characterization of Au@pNIPAM (red), Au/Pt@pNIPAM (orange), Au/Pt/Ni 4 nm@pNIPAM (light blue) and Au/Pt/Ni 8 nm@pNIPAM (dark blue) colloids, as those shown in the TEM image in B). C) TEM-XEDS RGB image where a Au core (modified with Pt) is surrounded by a Ni shell. D) Hysteresis loops Au-Pt/Ni@pNIPAM colloids at 5 and $300 \mathrm{~K}$ showing ferromagnetic behavior.

Another example of a complex, multifunctional system is shown in Figure 6 [32]. Au NPs were first coated with a thin Pt shell, which was then used as a catalyst to grow metallic nickel. Figure 6A shows the UV-vis spectra of Au@pNIPAM and Au/Pt@pNIPAM characterized by a narrow plasmon peak, whereas in the core/shell Au-Pt/Ni@pNIPAM the LSPR band is broadened but still clearly visible. The overall core-shell structure can be easily discerned in TEM (Figure 6B), and the detailed composition of the metallic core is revealed by elemental mapping (Figure 6C). Being the optical response governed by the Au core, the extra functionality in these systems arises from the magnetic behavior of the $\mathrm{Ni}$ (eventually NiO) shell, which is demonstrated by the hysteresis curve indicating a soft ferromagnet with low coercivity and widened hysteresis loop at lower 

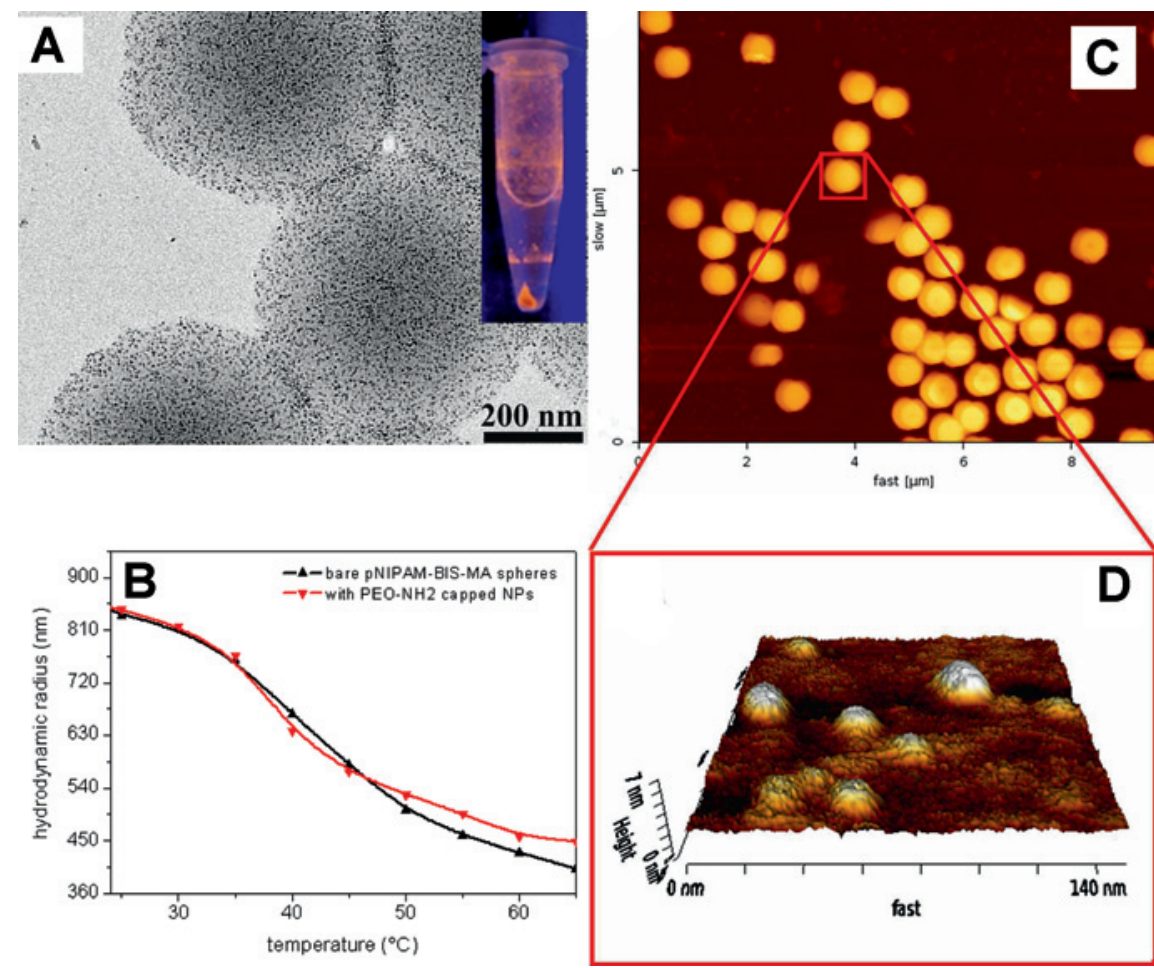

Figure 7: A) TEM image of pNIPAM microgels decorated with semiconductor CdSe/CdS NPs. The vial in the inset indicates that all semiconductor NPs and beads sediment together upon centrifugation. B) Hydrodynamic radius as a function of the temperature of bare pNIPAM beads (black) and those decorated with CdSe/CdS NPs (red). C) AFM topographic image of a collection of beads and surface topography of a single bead (D), showing protrusions with sizes comparable to those of the semiconductor NPs.

temperature. Again, the magnetic component allows further manipulation of the composites by applying an external magnetic field.

The versatility of microgels provides also the possibility to combine them with luminescent materials. In this context, fluorescent co-monomers can be copolymerized into the polymer network, allowing for confocal fluorescence microscopy imaging [33]. Fluorescent energy transfer studies have been performed during the collapse of pNIPAM-based core-shell systems containing donor and acceptor fluorophores, respectively [34]. Compared to metallic or magnetic NPs, fewer studies have been reported on the combination of pNIPAM with semiconductor NPs (colloidal quantum dots), which display tunable optical properties governed by quantum confinement [35]. The reason for this might be the frequent luminescent 
quenching upon small changes of the semiconductor NPs surface. There are, however, several examples of successful inclusion of quantum dots in microgels while maintaining luminescent properties [36-41], even when they are present in-situ, during the monomer polymerization [42].

Semiconductor NPs can also be immobilized on the surface of pNIPAM microgels, either covalently [17] (which may also yield variations of the fluorescence above and below LCST) or electrostatically [43]. An example of the latter is shown in Figure 7A, where a TEM image of CdSe/CdS core/shell semiconductor NPs capped with amino-terminated PEO ligands decorate the surface of pNIPAM beads copolymerized with maleic acid [43]. Figure 7B shows the variation of the hydrodynamic radius with temperature for pNIPAM beads before and after the incorporation of PEO-capped NPs. The hydrodynamic diameter does not vary significantly below the LCST but shows a slight increase for loaded spheres in the collapsed state, indicating the accommodation of the semiconductor NPs and ligands (PEO-derivatives) on the surface of the shallow beads, and an increased stiffness of the composite bead upon collapse. Further characterization of the composites was carried out by means of atomic force microscopy (AFM), as shown in Figure 7C,D. The heights of the protrusions visible in Figure 7D are $3 \mathrm{~nm}$ in average, close to the $4.5 \mathrm{~nm}$ size of the semiconductor NPs, which points toward a shallow inclusion in the polymeric network. Since the capping ligand shell of the NPs controls the interaction with the microgel, it is also possible to combine both semiconductor and metallic NPs on the surface of a pNIPAM bead, and to study the interaction between them below and above LCST [44]. Above LCST the average distance between NPs is reduced and the resulting interaction has been studied by optical spectroscopy and by optical trapping.

\section{Optical trapping of pNIPAM microbeads}

Optical trapping consists of using one or two highly focused laser beams to generate optical forces that can be used to spatially manipulate micro- and nanoobjects [45]. The setup is usually referred to as optical tweezers. The optical trapping setup is a force sensor calibrated from the principle that light momentum conservation provides force measurements that are independent of the size, shape, or refractive index of the trapped object [45]. Fitting the equilibrium power spectral density of force fluctuations of a particle in the trap allows acquiring the size of the trapped specimen [45]. Optical trapping has been mainly applied to study individual biological systems (cell, DNA, etc.) and individual dielectric objects, but semiconductor and even metallic particles of certain sizes can also be 

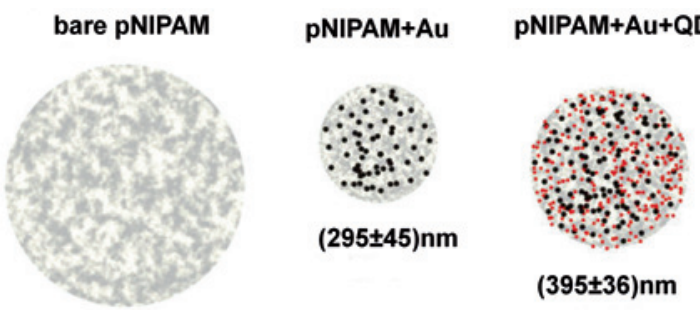

(395 \pm 36$) \mathrm{nm}$

(596士296)nm

$(906 \pm 98) \mathrm{nm}$
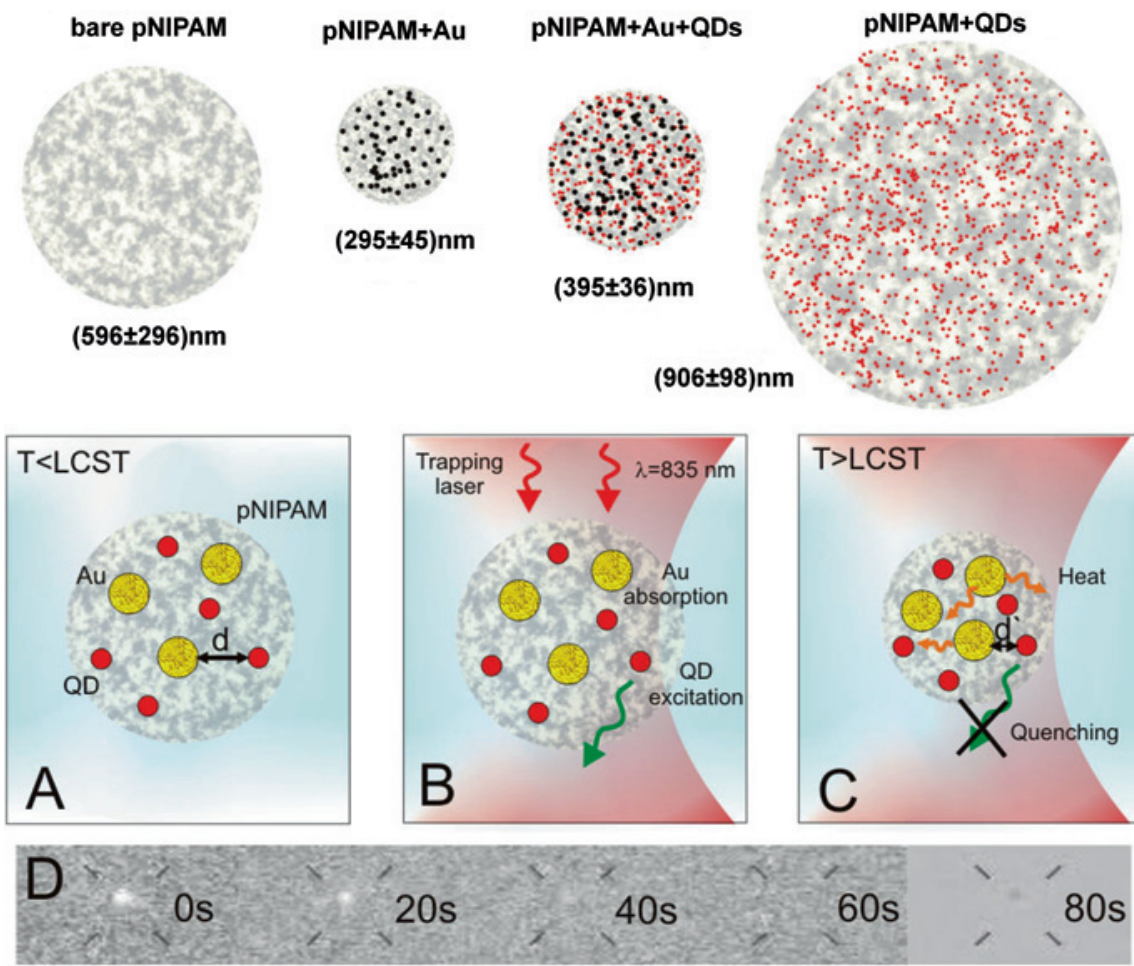

Figure 8: Comparative illustration of the average hydrodynamic sizes obtained for optically trapped bare pNIPAM microgels and the same microgels decorated with Au NPs, CdSe/CdS NPs or a combination of both. Quenching of pNIPAM covered with Au and CdSe/CdS NPs: (A) Initially, $T<$ LCST and $d$ denotes the average distance between NPs on the surface of the pNIPAM in this state. (B) When the pNIPAM/Au/QDs microgel bead is in the optical trap, NIR light is absorbed by $\mathrm{Au}$ and $\mathrm{CdSe} / \mathrm{CdS}$ NPs, hence emitting heat and fluorescence, respectively. (C) Heat emission leads to $T>$ LCST and subsequent pNIPAM shrinkage. $d^{\prime}$ denotes the average distance between Au and CdSe/CdS NPs which decreases in these conditions giving rise to luminescence quenching. (D) Snapshots of the quenching process for a single bead in the optical trap obtained by video-microscopy. Time of residence in the optical trap is indicated. See [44] for further details.

trapped. Experiments with microgels have been recently reported using an optical tweezers setup composed of two $835 \mathrm{~nm}$ lasers brought to the same focus. An automated hydrodynamic flow system allowed introduction of bare pNIPAM beads as well as beads decorated with Au NPs (see Figure 3A), with CdSe/CdS NPs (Figure 7A), or a combination of both [44]. Power spectrum analysis for individually manipulated specimens allowed the determination of size distributions for the different pNIPAM microgels (Figure 8 ). The method yields average diameters that 
are significantly affected by the presence of $\mathrm{Au}$ or $\mathrm{CdSe} / \mathrm{CdS}$ NPs, thus manifesting underlying light-NPs interaction effects. The average diameter of bare pNIPAM was determined to be $596 \pm 262 \mathrm{~nm}$, while bigger average diameters were found for microgels decorated with CdSe/CdS quantum dots, $d=906 \pm 98 \mathrm{~nm}$, suggesting that the NPs on the pNIPAM surface may reduce the heating effect of water in the interior of the microsphere and subsequent pNIPAM shrinkage. However, the average diameter obtained for pNIPAM covered with Au NPs was $295 \pm 45 \mathrm{~nm}$, which reveals that heating was induced by the thermal relaxation of excited LSPRs in the Au NPs present in the trap. The irradiation of metallic NPs increases the temperature of pNIPAM well above its LCST, even though the excitation laser was far from the Au NPs LSPR, where a maximum effect could be expected. Collective effects may thus be expected from the high concentration of Au NPs on the surface. Finally, the average diameter of the microgels containing both types of NPs was $395 \pm 36 \mathrm{~nm}$, i.e. smaller than pNIPAM exclusively covered with CdSe/CdS NPs but larger than microgels decorated with Au NPs only. These results emphasize the sensing capability of the system toward external temperature changes. Figure $8 \mathrm{~A}-\mathrm{C}$ illustrates the shrinkage of the microspheres upon trapping along with the luminescence quenching effect. Figure 8D shows several snapshots corresponding to the luminescent signal at different times $(0,20,40$, and $60 \mathrm{~s})$ of a single trapped microgel in the optical tweezers setup. In these images, the laser and the illumination signals were properly filtered except for the last frame on the right, which was recorded with unfiltered illumination light. As can be appreciated in this frame, the dark spot confirms that the microsphere remained in the trap after the luminescence intensity had fallen below the detection limit of the CCD camera (after approximately $1 \mathrm{~min}$ ), thus indicating that luminescence vanishes due to quenching. Induced heating by Au NPs induces extra shrinkage of the pNIPAM bead, and a subsequent approach of CdSe/CdS NPs to Au NPs quencher centers. This was also proven by steady state fluorescence spectroscopy in [44].

As mentioned above, metallic NPs absorb light and subsequently release heat. Therefore, Au NPs have been extensively studied in nanoscale thermometry, i.e. temperature measurements by nanometric objects that provide information from individual entities, such as cells. In this context, the characterization of single nanostructures allows understanding and controlling processes at the nanoscale such as membrane or DNA melting, drug release or protein denaturation [46-49]. Nanoscale thermodynamics of a single Au NP heated by a laser source has gained attention from both experimental and theoretical points of view [50-52]. Nanoscale thermometry of Au NPs has been addressed by Raman spectroscopy [53], time-resolved X-ray spectroscopy [54] or by photo-acoustic experiments [55]. Fluorescent molecules [56], semiconductor NPs [57, 58] and rare- 
earth compounds [59] exhibiting temperature-dependent spectra have also been used to estimate the temperature around excited Au NPs.

In order to account for temperature profiles within NPs, optical trapping experiments have also been performed. The configuration of Au@pNIPAM systems renders them ideal for nanoscale thermometry, as temperature changes can be studied in the vicinity of a single optically trapped Au NP giving rise to changes in the thermoresponsive material, both in refractive index and size. To this aim, changes in the plasmon resonance upon laser excitation have been determined in experiments with individual Au@pNIPAM NPs trapped by singlebeam optical tweezers $[60,61]$, and similar experiments were also reported to account for the temperature profile around an individual NP [46, 62]. An alternative way to understand the heat released by a single Au NP is by measuring the size of the trapped specimen, similarly to the experiments shown in Figure 8. A sketch of Au@pNIPAM NPs trapped in between two laser beams is shown in Figure 9A. Understanding the differences between data obtained from an ensemble of Au@pNIPAM NPs (using dynamic light scattering, DLS) and an individual particle (from optical trapping experiments) required a detailed analysis. Data obtained by DLS can be fitted to a four-parameter sigmoidal function (not shown) [63]. Taking into account the temperature predictions in light trapping experiments for different laser powers [64] the equation shown in Figure 9 can be obtained. This equation predicts the final size of a stratified Au@pNIPAM sphere, as a function of the optical power in the focus of the laser trap, for a range of laser powers inducing the pNIPAM phase transition. The size of the Au@pNIPAM system was found to change with the laser power, indicating that there is no direct correlation between laser power and surface temperature, as they are nonlinearly dependent. The combination of bulk DLS measurements with the temperature predictions in light trapping measurements provided a model to account for single-particle laser-induced Au@pNIPAM shrinkage. Figure 9B shows a comparison between sizes obtained by optical trapping and size predictions according to the equation. The black dots are particle size measurements obtained from optical trapping, where corrections of water viscosity with temperature were taken into account [63]. Solid lines correspond to the sigmoidal function in the vicinity of the temperature-induced pNIPAM phase transition. The dashed lines are linearized curves (see [63] for further details).

From Figure 9B it can be seen that a reasonable good agreement was found between DLS of and optical trapping, especially at low laser powers. However, size discrepancies are apparent as the laser power increases, which were assigned to a radial temperature decay within the pNIPAM shell (as sketched in Figure 9A), so that in pNIPAM layers that are farther away from the Au core temperature increases less and the contraction is smaller. In contrast, in the DLS 

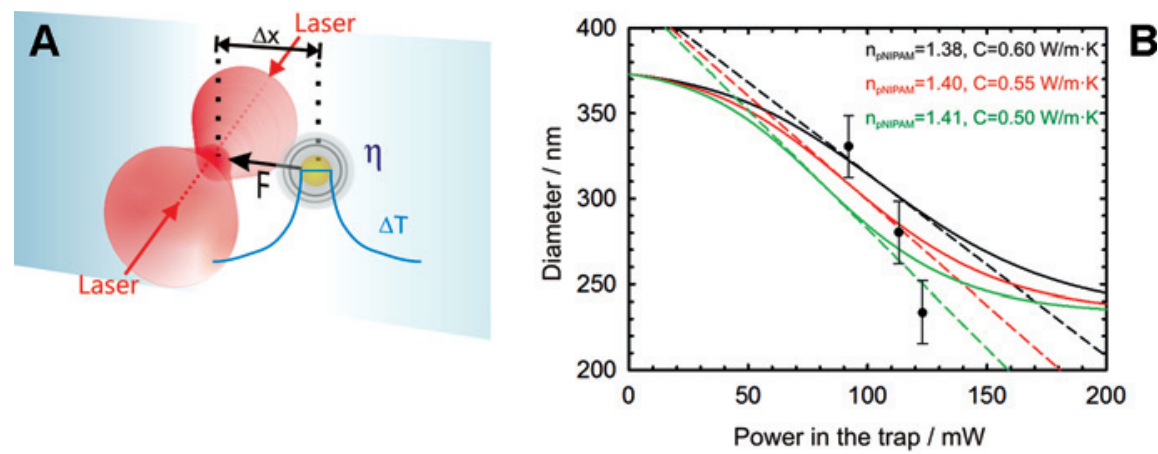

$$
D_{f}=D_{\text {min }}+\frac{1}{2}\left(1+\frac{T_{\text {tran }}-T_{\infty}}{2 \tau}\right)\left(D_{\text {max }}-D_{\text {min }}\right)-\frac{Q_{a b s} P_{\text {trap }}}{8 \pi C \tau} \frac{a_{c}^{2}}{W_{0}^{2}} \ln \frac{D_{\text {max }}}{D_{\min }}
$$

Figure 9: A) Au@pNIPAM hybrid system trapped in optical tweezers, where the $\Delta T$ curves represent the temperature profile from the inner core to the outer shell. The equation predicts the final size as a function of laser power in optical trapping experiments. $D_{\mathrm{f}}$ is the diameter of the composite, $D_{\min }$ and $D_{\max }$ are the minimum and maximum diameters, respectively, $T_{\text {tran }}$ is the midpoint transition temperature, and $2 \tau$ is the half-width of the transition. $a_{\mathrm{c}}$ is the radius of the Au core, $W_{0}$ is the Gaussian beam width and $P_{\text {trap }}$ is the optical power in the focal region, $C$ is the thermal conductivity of water and $Q_{a b s}$ is the absorption efficiency of the Au core immersed in pNIPAM/water. B) Comparison of sizes obtained from optically trapped Au@pNIPAM (black dots) and predicted by the equation. Three sets of parameters are represented with different colors to account for the influence of $C$ and refractive index variations during laser heating $\left(n_{\mathrm{pNIPAM}}\right)$. The solid lines correspond to the integrated sigmoidal function, and the dashed line to integrated linear behavior in the vicinity of the pNIPAM temperature-induced phase transition $\left(T \approx T_{\text {tran }}\right)$. See [63] for further details.

experiments, all the pNIPAM layers are in thermal equilibrium and contract homogeneously with temperature. This difference implies variations in absorption efficiency of Au@pNIPAM particles and in thermal conductivity of the medium around $\mathrm{Au} \mathrm{NPs,} \mathrm{in} \mathrm{a} \mathrm{radial} \mathrm{fashion.} \mathrm{In} \mathrm{order} \mathrm{to} \mathrm{account} \mathrm{for} \mathrm{these} \mathrm{variations,}$ three sets of parameters (thermal conductivity, $C$ and refractive index, $n$ ) are represented in different colors in Figure 9B. Middle red lines correspond to npNI$\mathrm{PAM}=1.40$ and $C=0.55 \mathrm{~W} / \mathrm{m} \mathrm{K}$ and bottom green lines to nPNIPAM $=1.41$ and $C=0.50 \mathrm{~W} / \mathrm{m} \mathrm{K}$. These two cases represent the tendency of the pNIPAM refractive index (n) to increase with temperature $[65,66]$ and that of the thermal conductivity of the Au NP environment (C) to decrease. The latter effect is expected because the thermal conductivity of the polymer is lower than that of water [6770]. Since water molecules are released from the polymer network upon heating, the average conductivity of the Au NP environment will be closer to that of the polymer for increasing temperature values. The error bars depicted for the dots 
in Figure 9B correspond to the size range representing a maximum size calculated with $\left(n_{\text {pNIPAM }}=1.38, C=0.60 \mathrm{~W} / \mathrm{m} \mathrm{K}\right)$ and a minimum size calculated with $\left(n_{\text {pNIPAM }}=1.41\right.$ and $\left.C=0.50 \mathrm{~W} / \mathrm{m} \mathrm{K}\right)$ for each laser power. It can be clearly observed that better agreement is found from both sets of data when taking into account variations in the refractive index and conductivity of the surrounding medium. These parameters must be taken into account when considering local laser heating experiments in aqueous solution at the nanoscale. It is also clear that moderate heating at optical traps allows control over the volume phase transition of the beads, which can be very advantageous for applications requiring temperature control at the nanoscale, such us targeted heat-induced cell death.

\section{Applications of pNIPAM microgels}

The studies described in this article show that new synthetic methodologies and sophisticated techniques for advanced characterization have been developed, opening room for new applications. An example comprises core-shell pNIPAMbased beads with sizes below $100 \mathrm{~nm}$ (nano-gels), loaded with small interfering RNA (SiRNA). It has been shown that these systems bioconjugated with peptides can carry out selective targeting and SiRNA delivery to ovarian cancer cells, thereby reducing gene expression [33]. The ability of analytes to diffuse through the micro- or nanogel layer allows for a vast palette of potential detection routes as described below. Arrays of microgels on substrates have been also used as optical lenses [71] and biosensors recording ligand-protein interactions. Another active field of research based on microgels loaded with NPs is catalysis. Pure, alloyed or multilayered metallic NPs@pNIPAM allow modulating the catalytic activity in a non-monotonous way, through variations in the porosity of the microgel when the temperature is varied [72-76]. Furthermore, by controlling the synthetic parameters, the diffusion of reactants toward the metal core surface can be tuned and thus the catalytic activity can be finely tuned. This effect has been recorded, for example, in the redox reaction of hexacyanoferrate (III) and borohydride taking place on the surface of Au NPs through either collapsed or swallowed Au@pNIPAM in which the pNIPAM shell controls the flow of reactants [77]. It was observed that the crosslinker density employed for the synthesis of pNIPAM ultimately governs the thermal response, allowing for a fine modulation of the catalytic activity.

Another interesting application for metallic NPs encapsulated in microgels is their use as platforms for surface enhanced Raman scattering (SERS), an optical spectroscopy technique in which the Raman scattering signal is greatly amplified 

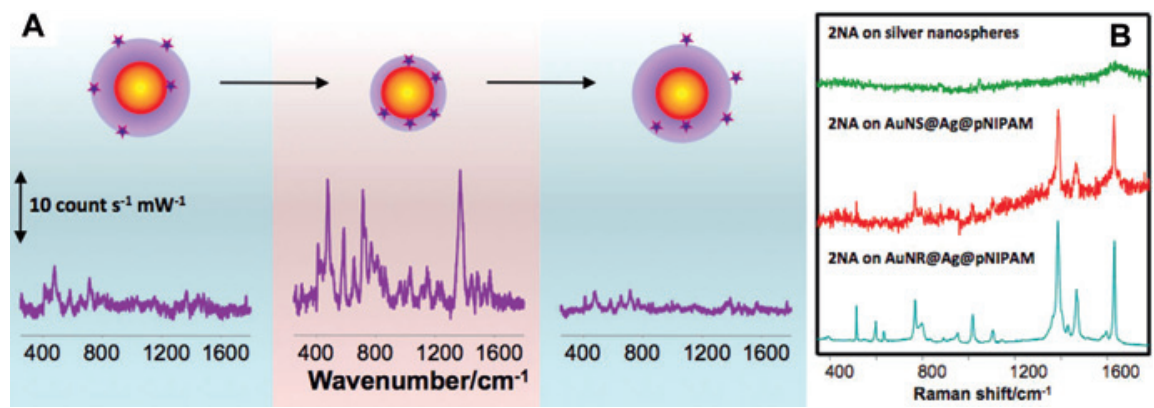

Figure 10: A) Variation of the SERS intensity of 1-naphthol as a function of the solution temperature (excitation wavelength, $785 \mathrm{~nm}$ ). B) SERS spectra of an alkaline solution of 2-naphthoic acid on different colloidal suspensions. Excitation laser line was $633 \mathrm{~nm}$. (100 and 82 in the name of the colloids refer to the sizes of the metallic cores).

in the vicinity of a nanostructured metal when it is excited in resonance with its localized surface plasmon [78, 79]. Since the first reported SERS on silver and gold colloids in 1979 [80], they have become one of the most commonly used nanostructures for SERS, both as a testing ground for the most thorough theoretical modeling, and for achieving single-molecule detection [81, 82]. The use of colloidal metals encapsulated with pNIPAM has demonstrated not only the amplification of the signals of molecules containing functional groups capable to interact with the surface of the metallic colloid but also those of neutral molecules that are difficult to detect such as alcohols or ethers. This effect can be seen in Figure 10A, where the SERS spectrum of 1-naphtol, a molecule with low affinity for Au surface, was recorded. Figure 10A shows the variation of SERS intensity as a function of solution temperature. The SERS signal can be identified after a swell-collapse transition, in which the pNIPAM corona traps the 1-naphtol molecules and brings them close enough to the metal surface for enhancement of the Raman signal.

Additionally, the amplification of the Raman signal can be optimized by varying the size, shape, and composition of the metallic core as well as the surface charge of the pNIPAM shell. On one hand, Ag-coated cores display improved plasmonic performance with respect to pure $\mathrm{Au}$ and on the other hand, the surface charge may allow electrostatic attraction of charged molecules. Therefore, Ag can be grown on the surface of Au NPs or Au nanorods embedded in pNIPAM by the reduction of $\mathrm{AgNO}_{3}$ with ascorbic acid, as described in Figure 5 above. Bimetallic cores embedded in pNIPAM shells synthesized using a cationic initiator yield composites with high positive surface charge. To demonstrate the possibility of using this system for electrostatic trapping, the SERS signal of 2-naphthoic acid (NA) at $\mathrm{pH} 13$ was recorded in different colloidal solutions namely: Ag nanospheres sta- 
bilized with citrate, AuAg bimetallic nanospheres with a positively charged pNIPAM shell (AuAgNS-100@pNIPAM) and AuAg bimetallic nanorods with Ag shell (AuAgNR-82@pNIPAM). Comparison of the SERS spectra can be seen in Figure 10B for 2-naphthoic acid at pH 13, so that NA is completely ionized and thus unlikely to adsorb on standard, citrate-stabilized silver colloids, which also have negative surface charge (see top spectrum Figure 10B). However, NA anions can adsorb on the positively charged shells of the metal@pNIPAM systems, giving rise to a significant increase in the recorded SERS intensity, as can be observed in both the middle and bottom spectra. A substantially larger intensity was recorded for nanorods, which is consistent with previous reports demonstrating the superior performance of this NP morphology for SERS analysis.

\section{Conclusions}

An overview of the fascinating properties of hybrid microgels has been presented, with an especial emphasis on particular combinations of inorganic NPs and pNIPAM microgels. The rational design of NPs and microgels allows the fabrication of a rich palette of responsive hybrid systems with tunable properties that can be applied in various fields including drug delivery, ultra-sensitive detection and catalysis, among others. It is however important to keep in mind that the intricate internal structure of microgels and the different size scales involved in these composites are of relevance when trying to understand their behavior and when devising their applications. In particular, the radial variations in porosity may affect the way in which drug delivery works or even the diffusion of reactant molecules and thus the performance of encapsulated catalyst nanoparticles or SERS enhancing substrates. Advances in the direction of such understanding have been made by means of single particle experiments such as optical manipulation and in situ characterization. Further progress is to be expected if full advantage is taken from multiple functionalities that can be incorporated in single nanoparticles as described in the present work.

Acknowledgement: This work was partly supported by the European Research Council (ERC Advanced Grant \#267867 Plasmaquo). 


\section{References}

1. Microgel Suspensions. Fundamentals and Applications, edited by: A. Fernandez-Nieves, H. M. Wyss, J. Mattsson, and D. A. Weitz, Wiley-VCH Verlag \& Co. KGaA (2011).

2. J. Cheng, B. A. Teply, I. Sherifi, J. Sung, G. Luther, F. X. Gu, E. Levy-Nissenbaum, A. F. Radovic-Moreno, R. Langer, and O. C. Farokhzad, Biomaterials 28 (2007) 869.

3. Y. W. Cho, S. A. Park, T. H. Han, D. H. Son, J. S. Park, S. J. Oh, D. H. Moon, K. J. Cho, C. H. Ahn, Y. Byun, I. S. Kim, I. C. Kwon, and S. Y. Kim, Biomaterials 28 (2007) 1236.

4. D. E. Owens and N. A. Peppas, Int. J. Pharm. 307 (2006) 93.

5. S. V. Vinogradov, Curr. Pharm. Des. 12 (2006) 4703.

6. W. H. Blackburn, E. B. Dickerson, M. H. Smith, J. F.McDonald, and L. A. Lyon, Bioconj. Chem. 20 (2009) 960.

7. M. Heskins and J. E. Guillet, J. Macromol. Sci. Chem. A2 (1968) 1441.

8. K. Kubota, S. Fujishige, and I. Ando, J. Phys. Chem. 94 (1990) 5154.

9. L. A. Lyon, Z. Y. Meng, N. Singh, C. D. Sorrell, and A. S. John, Chem. Soc. Rev. 38 (2009) 865.

10. S. Nayak and L. A. Lyon, Angew. Chem. Int. Ed. 44 (2005) 7686.

11. T. Tanaka and D. Fillmore, J. Chem. Phys. 70 (1979) 1214.

12. T. Tanaka, D. Fillmore, S. T. Sun, I. Nishio, G. Swislow, and A. Shah, Phys. Rev. Lett. 45 (1980) 1636.

13. Z.Hu, X. Lu, and J. Gao, Adv. Mater. 13 (2001) 1708.

14. L. S. Zha, Y. Zhang, W. L. Yang, and S. K. Fu, Adv. Mater. 14 (2002) 1090.

15. S. Nayak, D. J. Gan, M. J. Serpe, and L. A. Lyon, Small 1 (2005) 416.

16. M. Das, H. Zhang, and E. Kumacheva, Ann. Rev. Mater. Res. 36 (2006) 117.

17. M. Agrawal, J. Rubio-Retama, N. E. Zafeiropoulos, N. Gaponik, S. Gupta, V. Cimrova, V. lesnyak, E. López-Cabarcos, S. Txavalas, R. Rojas-Reyna, A. Eychmueller, and M. Stamm, Langmuir 24 (2008) 9820.

18. R. Contreras-Cáceres, A. Sánchez-Iglesias, M. Karg, I. Pastoriza-Santos, J. Pérez-Juste, J. Pacífico, T. Hellweg, A. Fernández-Barbero, and L. M. Liz-Marzán, Adv. Mater. 20 (2008) 1666.

19. X. Huang, P. K. Jain, I. H. El-Sayed, and M. A. El-Sayed, Nanomedicine 2 (2007) 681.

20. Y. Lu, Y. Mei, M. Ballauf, and M. Drechsler, J. Phys. Chem. B, 110 (2006) 3939.

21. Special issue on Synthesis and Plasmonic Properties of Nanostructures, MRS Bull. 30 (2005) 5.

22. M. Karg, I. Pastoriza-Santos, J. Pérez-Juste, T. Hellweg, and L. M. Liz-Marzán, Small 3 (2007) 1222.

23. M. Krag, Y. Lu, E. Carbó-Argibay, I. Pastoriza-Santos, J. Pérez-Juste, L. M. Liz-Marzán, and T. Hellweg, Langmuir 25 (2009) 3163.

24. M. S. Nikolic, M. Krack, V. Aleksandrovic, A. Kornowski, S. Foerster, and H. Weller, Angew. Chem. Int. Ed. 45 (2006) 6577-6580.

25. R. Contreras-Cáceres, J. Pacifico, I. Pastoriza-Santos, J. Pérez-Juste, A. Fernández-Barbero, and L. M. Liz-Marzán, Adv. Funct. Mater. 19 (2009) 3070.

26. M. Karg, S. Jaber, T. Hellweg, and P. Mulvaney, Langmuir 27 (2011) 820.

27. C. Fernández-López, C. Prez-Balado, J. Pérez-Juste, I. Pastoriza-Santos, A. R. de Lera, and L. M. Liz-Marzan, Soft Matter 8 (2012) 4165. 
28. F. Sauzedde, A. Elaïssari, and C. Pichot, Colloid Polym. Sci. 277 (1999) 846.

29. J. Guo, W. L. Yang, YH Deng, CC Wang, and S. Fu, Small 1 (2005) 737.

30. R. Contreras-Cáceres, S. Abalde-Cela, P. Guardia Giros, A. Fernández-Barbero, J. PérezJuste, R. A. Álvarez-Puebla, and L. M. Liz-Marzan Langmuir 27 (2011) 4520.

31. R. Contreras-Cáceres, I. Pastoriza-Santos, R. A. Álvarez-Puebla, J. Pérez-Juste, A. Fernandez-Barbero and L. M. Liz-Marzan, Chem. Eur. J. 16 (2010) 9462.

32. A. Sanchez-Iglesias, M. Grzelczak, B. Rodriguez-González, P. Guardia-Girós, I. PastorizaSantos, J. Pérez-Juste, M. Prato, and L. M. Liz-Marzán, ACS Nano 3 (2009) 3184.

33. W. H. Blackburn, E. B. Dickerson, M. H. Smith, J. F. McDonald, and L. A. Lyon, Bioconj. Chem. 20 (2009) 960.

34. D. Gan and L. A. Lyon, J. Am. Chem. Soc. 123 (2001) 8203.

35. Andrey L. Rogach Semiconductor Nanocrystal Quantum Dots. Synthesis, Assembly, Spectroscopy and Applications. Springer-Wien-New York (2008).

36. J. G. Zhang, S. Q. Xu and E. Kumacheva, J. Am. Chem. Soc. 126 (2004) 7908.

37. J. G. Zhang, S. Q. Xu, and E. Kumacheva, Adv. Mater. 17 (2005) 2336.

38. D. Suzuki and H. Kawaguchi, Langmuir 22 (2006) 3818.

39. D. Janczewski, N. Tomczak, M. Y. Han, and G. J. Vancso, Macromolecules 42 (2009) 1801.

40. Y. J. Gong, M. Y. Gao, D. Y. Wang, and H. Mohwald, Chem. Mater. 17 (2005) 2648.

41. M. Kuang, D. Y. Wang, H. B. Bao, M. Y. Gao, H. Mohwald, and M. Jiang, Adv. Mater. 17 (2005) 267.

42. W. Sheng, S. Kim, J. Lee, S. W. Kim, K. Jensen, and M. G. Bawendi, Langmuir 22 (2006) 3782.

43. A. Salcher, M. S. Nikolic, S. Casado, M. Vélex, H. Weller, and B. H. Juárez, J. Mater. Chem. 20 (2010) 1367.

44. S. Hormeño, N. G. Bastús, A. Pietsch, H. Weller, J. R. Arias-González, and B. H. Juárez, Nano Lett. 11 (2011) 4742.

45. S. B. Smith, Y. Cui, and C. Bustamante, Methods Enzymol. 361 (2003) 134.

46. P. M. Bendix, S. N. S. Reihani, and L. B. Oddershede, ACS Nano 4 (2010) 2256.

47. Z. Qin and J. C. Bischof, Chem. Soc. Rev. 41 (2012) 1191.

48. P. Haro-Gonzalez, W. T. Ramsay, L. M. Maestro, B. del Rosal, K. Santacruz-Gomez, M. C. Iglesias-de la Cruz, F. Sanz-Rodriguez, J. Y. Chooi, P. R. Sevilla, M. Bettinelli, D. Choudhury, A. K. Kar, J. García Solé, D. Jaque, and L. Paterson, Small 9 (2013) 2162.

49. J. Do, R. Schreiber, A. A. Lutich, T. Liedl, J. Rodriguez-Fernandez, and J. Feldmann, Nano Lett. 12 (2012) 5008.

50. H. Goldenberg and C. J. Tranter, British J. Appl. Phys. 3 (1952) 296.

51. V. K. Pustovalov, Chem. Phys. 308 (2005) 103.

52. V. K. Pustovalov and V. A. Babenko, Laser Phys. Lett. 1 (2004) 516.

53. H. H. Richardson, Z. N. Hickman, A. O. Govorov, A. C. Thomas, W. Zhang, and M. E. Kordesch, Nano Lett.6 (2006) 783.

54. A. Siems, S. A. L. Weber, J. Boneberg, and A. Plech, New J. Phys. 13 (2011) 043018.

55. J. Shah, S. Park, S. Aglyamov, T. Larson, L. Ma, K. Sokolov, K. Johnston, T. Milner, and S. Y. Emelianov, J. Biomed. Opt. 13 (2008) 034024.

56. G. Baffou, M. P. Kreuzer, F. Kulzer, and R. Quidant, Opt. Express 17 (2009) 3291.

57. A. Gupta, R. S. Kane, and D.-A. Borca-Tasciuc, J. Appl. Phys. 108 (2010) 064901.

58. L. M. Maestro, P. Haro-Gonzalez, J. G. Coello, and D. Jaque, Appl. Phys. Lett. 100 (2012) 201110. 
59. M. L. Debasu, D. Ananias, I. Pastoriza-Santos, L. M. Liz-Marzan, J. Rocha, and L. D. Carlos, Adv. Mater. 25 (2013) 4868.

60. C. M. Pitsillides, E. K. Joe, X. Wei, R. R. Anderson, and C. P. Lin, Biophys. J. 84 (2003) 4023.

61. J. Rodriguez-Fernandez, M. Fedoruk, C. Hrelescu, A. A. Lutich, and J. Feldmann, Nanotechnology 22 (2011) 245708.

62. F. Sanz-Rodriguez, J. Y. Chooi, P. R. Sevilla, M. Bettinelli, D. Choudhury, A. K. Kar, J. García Solé, D. Jaque, and L. Paterson, Small 9 (2013) 2162.

63. S. Hormeño, P. Gregorio-Godoy, J. Pérez-Juste, L. M. Liz-Marzán, B. H. Juárez, and J. R. Arias-Gonzalez, Small 10 (2014) 376.

64. W. Andra, C. G. d’Ambly, R. Hergt, I. Hilger, and W. A. Kaiser, J. Magn. Magn. Mater. 194 (1999) 197.

65. B. W. Garner, C. Tong, S. Ghosh, Z. Hu, and A. Neogi, Appl. Phys. Express 2 (2009) 057001.

66. S. Schmidt, H. Motschmann, T. Hellweg, and R. von Klitzing, Polymer 49 (2008) 749.

67. O. Andersson and G. P. Johari, J. Chem. Phys. 134 (2011) 124903.

68. F. Arai, C. Ng, H. Maruyama, A. Ichikawa, H. El-Shimy, and T. Fukuda, Lab Chip 5 (2005) 1399.

69. J. M. P. Coelho, M. A. Abreu, and F. Carvalho Rodrigues, Opt. Lasers Eng. 42 (2004) 27.

70. S. R. Davidson and M. D. Sherar, Int. J. Hyperthermia 19 (2003) 551.

71. J. Kim, M. J. Serpe, and L. A. Lyon, J. Am. Chem. Soc. 126 (2004) 9512.

72. M. Schrinner, S. Proch, Y. Mei, R. Kempe, N. Miyajima, and M. Ballauff, Adv. Mater. 20 (2008) 1928.

73. Y. Lu, Y. Mei, M. Schrinner, M. Ballauff, M. W. Moller, and J. Breu, J. Phys. Chem. C 111 (2007) 7676.

74. J. Je, T. Huynh, Y. Hu, and Y. Yin, Nano Lett. 8 (2008) 931.

75. Y. Lu, Y. Mei, Y, M. Drechsler, and M. Ballauff, Angew. Chem., Int. Ed. 45 (2006) 813.

76. Y. Lu, Y. Mei, M. Drechsler, and M. Ballauff, J. Phys. Chem. B 110 (2006) 3930.

77. S. Carregal-Romero, N. J. Buurma, J. Pérez-Juste, L. M. Liz-Marzán, and P. Hervés, Chem. Mater. 22 (2010) 3051.

78. R. A. Álvarez-Puebla, R. Contreras-Cáceres, I. Pastoriza-Santos, J. Pérez-Juste, and L. M. Liz-Marzán, Angew. Chem., Int. Ed. 48 (2009) 138.

79. R. Contreras-Cáceres, I. Pastoriza-Santos, R. A. Álvarez-Puebla, J. Pérez-Juste, A. Fernández-Barbero, and L. M. Liz-Marzán, Chem. Eur. J. 16 (2010) 9462.

80. J. A. Creighton, C. G. Blatchford, and M. G. Albretch, J. Chem. Soc. Faraday Trans. 275 (1979) 790.

81. K. Kneipp, Y. Wang, H. Kneip, L. T. Perelman, I. Itzkan, R. R. Dasari, and M. Feld, Phys. Rev. Lett. 78 (1997) 1667.

82. S. Nie and S. R. Emory, Science 275 (1997) 1102. 